\title{
Um Modelo para Avaliação da Qualidade da Gerência de Projetos de Software
}

\author{
Eliseu Castelo Branco Júnior \\ eliseu@acm.org \\ Arnaldo Dias Belchior (Orientador) \\ belchior@unifor.br \\ Universidade de Fortaleza (UNIFOR) \\ Mestrado de Informática Aplicada (MIA / CCT) \\ Av. Washington Soares, 1321, Edson Queiroz \\ CEP 60.811-341 - Fortaleza-CE, Brasil
}

\begin{abstract}
Resumo
Este trabalho desenvolve um modelo para avaliar a qualidade de processos gerenciais de software, considerando as áreas de gerência do $\mathrm{PMBOK}$, com suas respectivas categorias de processos, em aderência à visão de qualidade de processos abordada no ISO/IEC TR 15504, em consonância com as normas ISO/IEC 12207, ISO 10006, e ISO/IEC DTR 16326. É apresentado o resultado de uma pesquisa de campo, realizada com gerentes de projetos e especialistas em computação, sobre a importância de processos gerenciais em projetos de software. Esses resultados foram obtidos e validados através de um modelo para a avaliação da qualidade de software.
\end{abstract}

Palavras-chave: qualidade de software, gerência de projetos, modelagem de processos.

\begin{abstract}
This study develops a model for the appreciation of the quality of software process management, considering PMBOK management areas with their respective process categories according to the visualization of process quality approached in ISO/IEC 15504 and in accordance with ISO/IEC 12207, ISO 10006, and ISO/IEC DTR 16326 rules. The results of a questionnaire are presented, which was carried out with project managers and computing specialists on the importance of software project management. Such results were obtained and validated by means of a model for the appreciation of software quality.
\end{abstract}

Keywords: software quality, project management, software process.

\section{Introdução}

Um projeto de software é uma atividade que tem início e fim delimitados, com objetivos estabelecidos dentro de parâmetros de escopo, custo, tempo e qualidade, risco, suprimentos, recursos humanos e comunicação. Normalmente, um produto gerado por um projeto de software deve atender às especificações feitas por quem financiou seu desenvolvimento e realizar os objetivos que lhes foram propostos.

A aplicação de técnicas de engenharia de software aos projetos é feita com o objetivo de reduzir custos, obter métricas quantitativas e qualitativas sobre o processo e disciplinar o trabalho das equipes de desenvolvimento [5]. Muitos dos problemas, que afetam os projetos de desenvolvimento de software, são de ordem gerencial e não técnicos. Esta afirmação vem 
de encontro ao mito de que os problemas, que causam falhas e atrasos no processo de produção de software, são de natureza técnica. A gerência de projetos de software vem suprir essas deficiências assumindo a função de coordenar a ação de pessoas, na execução de processos de uma forma disciplinada e conseqüente [17].

Este trabalho descreve uma metodologia para a avaliação da execução da gerência de processos em projetos de software. Seja qual for a natureza do projeto de software, os objetivos estabelecidos para esse projeto reportar-se-ão a uma das áreas de gerenciamento utilizadas. Propõe-se, então, que a avaliação da qualidade dos processos gerenciais seja feita a partir de documentos gerenciais produzidos pelo projeto.

Os objetivos específicos a que se propõe este trabalho são os seguintes:

- fornecer aos gerentes de projetos uma ferramenta para avaliação, que contenha informações sobre os pontos fortes e fracos do projeto, indicando os problemas que poderão surgir pela aplicação incorreta ou pela não aplicação dos processos de planejamento, execução e controles adequados no projeto;

- divulgar os processos reconhecidos internacionalmente como sendo os mais relevantes na gestão eficaz de projetos;

- enfatizar a importância do planejamento e das ações preventivas na gestão de projetos.

O modelo proposto poderá ser utilizado como uma ferramenta de apoio aos gerentes de projetos de software nas seguintes atividades:

- definição de processos de gerenciamento padronizados para projetos de software;

- hierarquização desses processos, segundo o grau de importância de cada um, no contexto da gerência ao qual está inserido (gerência de custo, de prazo, de qualidade, etc.);

- avaliação da qualidade de trabalhos de gerenciamento de projetos de software, de acordo com um padrão de qualidade obtido em uma pesquisa de campo;

- melhoria dos fatores de prazo, custo e qualidade dos trabalhos realizados pela equipe do projeto.

Este trabalho está organizado da seguinte forma: além desta introdução, na seção 2, Processos de Software, apresentam-se normas de qualidade de processos e modelos de processos de software, relacionando-os com a gerência de projetos de software. Na seção 3, Gerência de Projetos, discorre-se sobre vários aspectos da gerência de projetos, enfocando-se os estudos do SWEBOK [19], PRINCE [18] e PMBOK [15]. Na seção 4, Avaliação da Qualidade de Projetos de Software, delineia-se uma metodologia para a avaliação da qualidade da gerência de projetos de software, que foi consolidada através de uma pesquisa de campo com gerentes de projetos e especialistas em engenharia de software. Na seção 5, Características de Qualidade da Gerência de Projetos de Software, os resultados da pesquisa de campo são apresentados e analisados em relação aos principais processos considerados para a avaliação da qualidade de projetos de software. Na seção 6, Conclusão, são apresentadas as conclusões e as contribuições deste trabalho.

\section{Processos de Software}

Para que se possa gerenciar de forma adequada projetos de software é necessário que se conheça suficientemente seu processo de desenvolvimento em suas mais variadas facetas. 
Um processo de software pode ser definido como um conjunto de atividades, métodos, práticas e transformações, que desenvolvedores utilizam para construir e manter software e produtos associados, como planos do projeto, documentação, código fonte e manuais [5, 6].

Em uma organização imatura, os produtos de software são geralmente produzidos por especialistas e seu gerenciamento é feito durante o andamento do projeto. Mesmo que uma etapa do processo seja especificada anteriormente, esta não é rigorosamente seguida. Nessas organizações, o gerenciamento está focado na solução de problemas imediatos (conhecido como "apagar incêndios"). Cronogramas e orçamentos são rotineiramente excedidos porque não se baseiam em estimativas realistas. Quando fortes restrições de tempo e recursos são impostas, a funcionalidade e a qualidade do produto são freqüentemente comprometidas, para atender ao cronograma imposto $[12,13]$. Não há bases objetivas para o julgamento da qualidade dos produtos gerados ou para a resolução de problemas do processo ou do produto de software. É difícil medir a qualidade de tais produtos, pois as atividades, que visam a sua melhoria, são muitas vezes eliminadas, para que o projeto não ultrapasse o cronograma previsto.

Em uma organização madura, a função do gerente é monitorar a qualidade do produto de software e a satisfação do cliente. Há objetivos definidos, bases quantitativas para julgamento da qualidade dos produtos de software e análise de problemas do processo. Orçamentos e cronogramas são baseados em resultados históricos de atuação; as expectativas de custo, cronograma, funcionalidade e qualidade são usualmente atingidas. A seguir, serão apresentadas algumas normas internacionais de qualidade, que apóiam a gerência de projetos.

A norma de gestão e garantia da qualidade, ISO 9000-3, contém diretrizes para a aplicação da ISO 9001:2000 para o desenvolvimento, o fornecimento e a manutenção de software, sendo utilizada para descrever controles e métodos sugeridos para a produção de software, que atendam aos requisitos estabelecidos pelo usuário [8].

A norma ISO/IEC 10006 Gestão da Qualidade - Diretrizes para a qualidade no gerenciamento de projetos tem como objetivo fornecer diretrizes sobre os elementos do sistema de qualidade, conceitos e práticas, para as quais a implementação é importante, tendo impacto na obtenção da qualidade no gerenciamento de projetos. Essa norma fornece também diretrizes sobre como a organização promotora deve aprender com o projeto, como parte de um programa para melhoria contínua em outros projetos, atuais e futuros.

A ISO/IEC 12207 estabelece uma estrutura comum para os processos de ciclo de vida de software, com terminologia bem definida para execução, gerenciamento e melhoria de software. A estrutura contém processos, atividades e tarefas a serem aplicados durante a aquisição, fornecimento, desenvolvimento, manutenção e operação de produtos de software e que devem ser adaptados ao contexto e às características de cada projeto de software. Essa adaptação consiste na exclusão de processos, atividades e tarefas não aplicáveis ao processo. Os processos devem estar em conformidade com as políticas e normas já existentes na organização e deve-se evitar conflitos com os procedimentos já estabelecidos [11].

O processo organizacional de gerência da norma ISO/IEC 12207 contém as atividades genéricas, que podem ser empregadas para o gerenciamento de projetos. Para prover os gerentes de projetos de um guia para aplicação da norma ISO/IEC 12207 em projetos de software, a ISO elaborou o relatório técnico ISO/IEC DTR 16326 - Guia para aplicação da ISO/IEC 12207 para a gerência de projetos. Esse guia relaciona a norma ISO/IEC $12207 \mathrm{com}$ as áreas de gerenciamento do PMBOK e com a norma ISO 10006 - Guia para qualidade no gerenciamento de projetos, conforme a Figura 1. 


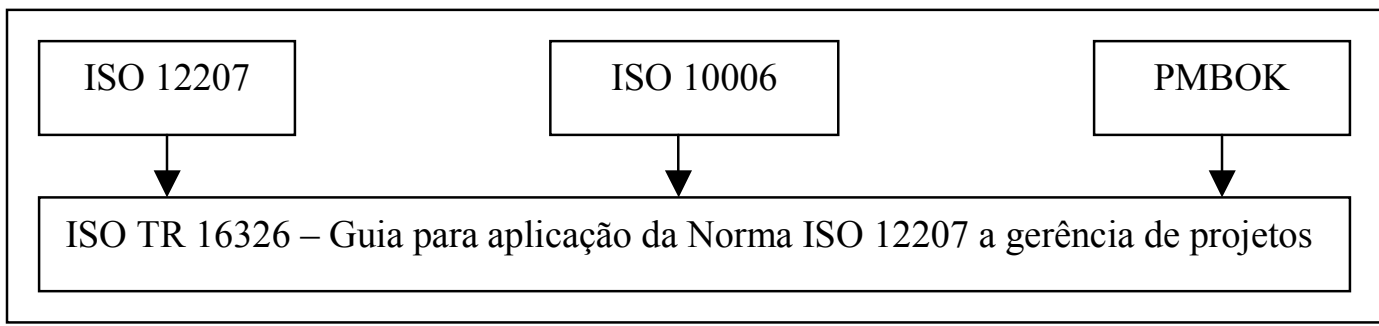

Figura 1: Uso da ISO/IEC 12207, PMBOK e ISO 10006 do ISO/IEC DTR 16326

Neste trabalho, a avaliação da qualidade de projetos de software foi realizada, considerando-se as normas ISO 12207 e ISO 10006 e os relatórios técnicos ISO/IEC TR 15504 e ISO/IE DTR 16326, juntamente com o PMBOK, que será apresentado na seção seguinte. Nessa avaliação, cada área de gerência de projetos considerada foi subdividida em suas respectivas categorias de processos. Essas categorias, por sua vez, foram compostas de seus processos gerenciais, que passaram por um processo de avaliação.

\section{Gerência de Projetos}

Projetos são freqüentemente efetuados com o objetivo de atingir as metas do plano estratégico da organização, enquanto que as tarefas operacionais executam as atividades rotineiras e repetitivas. O projeto pode ser definido em termos de suas características específicas: um projeto é um esforço temporário, que visa à criação de um produto ou serviço exclusivo. Temporário significa que cada projeto possui data de início e de conclusão bem definidas. Produto ou serviço exclusivo significa que o produto ou serviço produzido é diferente de alguma maneira de todos os outros produtos ou serviços elaborados pela organização.

O contexto do gerenciamento de projetos nas organizações pode ser influenciado pelos seguintes fatores [15]:

- o modelo do ciclo de vida escolhido para o projeto;

- a equipe envolvida direta ou indiretamente com o projeto;

- a cultura organizacional da empresa;

- a cultura dos membros da equipe, especialmente, quando constituída por indivíduos de países diferentes;

- questões sócio-econômicas tais como normas, padrões e regulamentos;

- o perfil gerencial do responsável pelo projeto, que deve deter conhecimentos sólidos, especialmente sobre finanças, planejamento estratégico, estrutura organizacional, recursos humanos, políticas e normas.

A gerência de projetos é a primeira camada do processo de engenharia de software, porque abrange todo o processo de desenvolvimento, do início ao fim. Devido à importância da gerência de projetos para o sucesso do projeto, todos os profissionais, que trabalham como gerentes de projetos, deveriam saber como colocá-la em prática. Infelizmente, muitos não a entendem [5].

Em projetos de software, tem-se firmado, cada vez mais, o entendimento de que a utilização de metodologias científicas vem de sobremaneira auxiliar no processo de 
gerenciamento. Neste contexto, serão apresentadas três importantes metodologias para o gerenciamento de projetos de software: PRINCE, SWEBOK e PMBOK.

PRINCE (PRojects IN Controlled Environments) é um método estruturado para melhorar a eficácia do gerenciamento de projetos de sistemas de informação, estabelecido pela CCTA (Central Computer and Telecommunications Agency). É um padrão que possui reconhecimento internacional e é utilizado intensivamente pelo governo britânico e por empresas do setor privado. Esse método é de domínio público, oferecendo orientações sobre as melhores práticas para o gerenciamento de projetos para as organizações [18].

As principais características do PRINCE são:

- foco no negócio;

- estrutura organizacional definida para a equipe de gerenciamento do projeto;

- planejamento baseado no produto;

- ênfase na divisão do projeto em fases gerenciáveis e controláveis;

- flexibilidade para ser aplicado às várias fases do projeto.

Este método melhora o gerenciamento e a condução de projetos através do uso controlado dos recursos e da habilidade de gerenciar os riscos do projeto e do negócio com maior eficácia. Provê uma linguagem comum para todos os participantes do projeto, encorajando o reconhecimento formal das responsabilidades das partes envolvidas. Mantém o foco nos resultados que o projeto deve alcançar, enfatizando porque, quando e para quem esses resultados devem ser entregues.

O SWEBOK (Guide to the Software Engineering Body of Knowledge) representa um esforço para organizar e catalogar um conjunto de conhecimentos sobre engenharia de software e proporcionar descrições completas, sistemáticas e concisas desta disciplina. Como essa disciplina é ainda jovem, embora sua estrutura já seja estabelecida, há um interesse especial em capturar e categorizar as sub-disciplinas, que a compõem. A classificação proposta no SWEBOK tem a intenção de identificar aqueles elementos que não variam, mesmo que novas tecnologias sejam incorporadas dentro de sua estrutura [16].

O PMBOK (Project Management Body of Knowledge), compilado pelo PMI (Project Management Institute), organiza áreas de conhecimento e de processos na prática do gerenciamento de projetos [16].

Esta metodologia decompõe um projeto em processos, que são ações realizadas para se obter um resultado específico. Esses processos são categorizados em processos de gerenciamento e processos de produção.

Os processos de gerenciamento de projetos são organizados nos cinco grupos abaixo, cada um contendo um ou mais processos [15]: Processos de iniciação: reconhecimento de que o projeto deve ser iniciado ou uma de suas fases, havendo a autorização para o início. Processos de planejamento: desenvolvimento e manutenção de um esquema de trabalho para o acompanhamento de demandas da empresa, que o projeto compromete-se a atender. Processos de execução: coordenação de pessoal e de recursos designados para a execução das atividades do projeto. Processos de controle: validação dos objetivos alcançados pelo projeto, através da monitoração e medida do progresso das atividades e aplicação de medidas corretivas, quando for necessário. Processos de finalização: aceitação formal dos produtos gerados pelo projeto ou por suas fases, e o encerramento do projeto ou de suas fases.

Os cinco grupos de processos do PMBOK estão ligados entre si pelo resultado que produzem. O produto de saída de um grupo torna-se produto de entrada para o outro grupo. 
Entre os dois grupos centrais (execução e controle) as ligações são de duplo sentido. Os processos de execução fornecem informações para os processos de controle, que podem prover informações de volta para modificar os processos de execução (Figura 2).

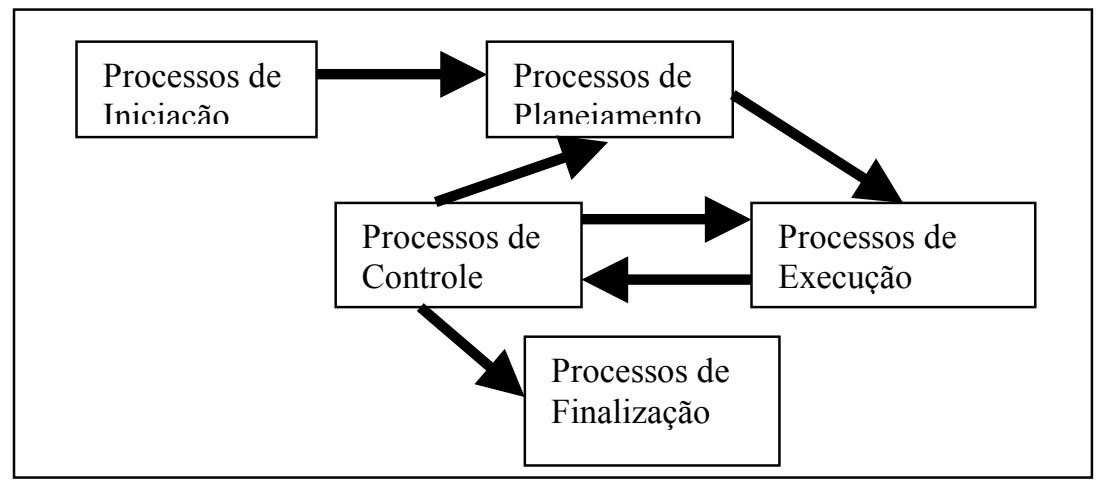

Figura 2: Ligações entre os grupos de processo em uma fase [15]

Dentro de cada grupo, os processos podem ser descritos em termos de:

- Entradas: documentos ou itens documentáveis, que serão produzidos no início do processo.

- Ferramentas e técnicas: mecanismos aplicados às entradas para gerar as saídas.

- Saídas: documentos ou itens documentáveis, que são o resultado do processo.

Alguns grupos de processos são classificados em processos essenciais e processos facilitadores. Os processos essenciais mantêm uma clara dependência entre si, devendo ser executados em uma determinada ordem na maioria dos projetos. Os processos facilitadores são dependentes da natureza do projeto, mantendo entre si uma dependência fraca. Apesar dessa característica flexível, não são opcionais.

Segundo o PMI, o conhecimento necessário para gerenciar projetos de software está distribuído em nove áreas, chamadas de áreas de gerência de processos. São elas as seguintes [20]:

1. Gerência da Integração: desenvolve e controla a execução do plano do projeto, gerenciando as mudanças, que ocorrem no projeto durante seu desenvolvimento.

2. Gerência do Escopo: abrange tarefas, atividades, contratos, responsabilidades, missões e atribuições, determinando as fronteiras entre os processos.

3. Gerência do Prazo: determina os prazos para cada atividade, o controle do cronograma do projeto, o seqüenciamento e a estimativa de duração dessas atividades.

4. Gerência de Custos: envolve o planejamento dos recursos a serem alocados ao projeto, a estimativa e o controle de custos, e a orçamentação.

5. Gerência da Qualidade: faz o planejamento, o controle e a garantia da qualidade.

6. Gerência de Recursos Humanos: engloba o planejamento da organização do projeto, a aquisição de pessoal e a capacitação da equipe.

7. Gerência de Comunicações: compõe-se do planejamento da comunicação, da disponibilização da informação, da comunicação da performance, e do fechamento administrativo. 
8. Gerência de Riscos: promove a identificação e a quantificação de riscos (danos físicos, riscos tecnológicos, saída de membros da equipe), o planejamento e controle de respostas a esses riscos.

9. Gerência de Suprimentos: controla os contratos e os fornecimentos de materiais e equipamentos.

A seguir, será apresentada uma metodologia para a investigação da relevância de documentos gerenciais produzidos em projetos de software.

\section{Avaliação da Qualidade de Projetos de Software}

A avaliação da qualidade do gerenciamento de projetos de software será feita utilizando um padrão de qualidade estabelecido a partir de uma pesquisa de campo realizada com engenheiros de software e gerentes de projetos de software.

Essa pesquisa mediu a importância de processos de gerenciamento de projetos propostos pelo PMBOK, que são considerados como fundamentais para o gerenciamento eficaz de projetos $[3,4,5]$. A escolha desses processos foi feita em consonância com as normas ISO/IEC TR 15504 [9], ISO/IEC 12207 [11], ISO 10006 [10], e ISO/IEC DTR 16326 [7]. O objetivo da pesquisa é avaliar o grau de importância que os gerentes de projetos atribuem aos documentos gerenciais que devem ser produzidos pelo projeto. Essa nota servirá para estabelecer qual o padrão de qualidade que é esperado para o grupo de processos que compõem as áreas de gerência de projetos.

Para a avaliação da qualidade da gerência de projetos de software, foram utilizadas sete das nove áreas de gerência de projetos do PMBOK (2000), que tem sua estrutura em conformidade com a ISO/IEC 12207, e que são enfatizadas no ISO/IEC TR 15504 [9]. Essas áreas de gerências são: integração, escopo, prazo, custos, qualidade, comunicação, e riscos, agregando vinte e nove processos, sendo 16 principais e 13 auxiliares.

As outras duas áreas de gerência de recursos humanos e de gerência suprimentos não foram consideradas neste trabalho, pois, normalmente, não estão diretamente relacionados com o gerente do projeto de engenharia de software. Em geral, estas atividades são executadas por áreas administrativas específicas da empresa: área de recursos humanos e área de recursos logísticos.

O processo de avaliação da qualidade de projetos de software foi realizado, utilizandose o Modelo Fuzzy para Avaliação da Qualidade de Software [1]. A teoria dos conjuntos fuzzy (difusos) tem sido utilizada na representação de modelos de raciocínios imprecisos [14], que possuem um papel essencial na tomada de decisões racionais em ambientes de incerteza [21].

As etapas do Modelo fuzzy para avaliação da qualidade dos processos de gerenciamento de projetos são descritas a seguir [1]:

Primeira Etapa: estabelecimento do objeto da avaliação, do conjunto de itens a ser avaliado e das instituições a serem pesquisadas.

A pesquisa de campo realizada colheu a opinião de 30 gerentes de projetos e especialistas em informática de diversas instituições dos estados do Ceará, Espírito Santo, Rio de Janeiro e Distrito Federal, para a avaliação de processos gerenciais em projetos de software. 
Os itens avaliados compuseram um questionário de 52 questões, abrangendo todos os documentos gerenciais produzidos pelos processos principais do PMBOK. Na Tabela 1, é mostrado um extrato desse questionário.

Tabela 1: Extrato de parte do questionário de avaliação de processos gerenciais

\begin{tabular}{|c|c|c|c|c|c|}
\hline Gerência: PRAZO & & & & & \\
\hline Grupo de Processos: PLANEJAMENTO & & & & & \\
\hline Processo: DESENVOLVIMENTO DO CRONOGRAMA & & & & & \\
\hline Processo de Avaliação: & & & alot & & \\
\hline 1. O cronograma de realização da lista de atividades foi planejado? & 0 & 1 & 2 & 3 & 4 \\
\hline $\begin{array}{l}\text { 2. O plano de gerenciamento das mudanças no cronograma foi } \\
\text { desenvolvido? }\end{array}$ & 0 & 1 & 2 & 3 & 4 \\
\hline Comentários: & & & & & \\
\hline
\end{tabular}

Segunda Etapa: obtenção do perfil dos especialistas.

O perfil de cada especialista foi obtido na pesquisa, através do Questionário de Identificação do Perfil do Especialista (QIPE), envolvendo a experiência do mesmo em gerência de projetos e seu grau de conhecimento em informática.

Terceira Etapa: determinação do grau de importância de cada item identificado na primeira Etapa.

O procedimento de investigação consistiu na elaboração de um questionário e na definição de técnicas para o manuseio do mesmo. O questionário foi aplicado e os graus de importância selecionados pelos especialistas foram convertidos para números fuzzy triangulares normais do tipo-LR, $\tilde{N}\left(a_{\mathrm{i}}, b_{\mathrm{i}}, c_{\mathrm{i}}\right)$, previamente delineados (Tabela 2 ).

Tabela 2: Números fuzzy normais triangulares para a avaliação da qualidade [1]

\begin{tabular}{|c|c|l|l|}
\hline Nota & $\begin{array}{c}\text { Números Fuzzy } \\
\text { Triangulares }\end{array}$ & \multicolumn{1}{|c|}{$\begin{array}{c}\text { Termo } \\
\text { Lingüístico }\end{array}$} & Interpretação \\
\hline $\mathbf{0}$ & $\tilde{N}_{1}=(0,0 ; 0,0 ; 1,0)$ & Total Ausência & $\begin{array}{l}\text { Indica de maneira absoluta que o critério está } \\
\text { ausente ou não possui nenhuma importância }\end{array}$ \\
\hline $\mathbf{1}$ & $\tilde{N}_{2}=(0,0 ; 1,0 ; 2,0)$ & Baixa Presença & $\begin{array}{l}\text { Indica um baixo grau de presença do critério, seja } \\
\text { por deficiência ou pouca importância do mesmo. }\end{array}$ \\
\hline $\mathbf{2}$ & $\tilde{N}_{3}=(1,0 ; 2,0 ; 3,0)$ & Moderada Presença & $\begin{array}{l}\text { Indica um grau de presença moderada (aceitável) } \\
\text { do critério. }\end{array}$ \\
\hline $\mathbf{3}$ & $\tilde{N}_{4}=(2,0 ; 3,0 ; 4,0)$ & Alta Presença & $\begin{array}{l}\text { Indica um alto grau de presença do critério, mas } \\
\text { não de forma plena. }\end{array}$ \\
\hline $\mathbf{4}$ & $\tilde{N}_{5}=(3,0 ; 4,0 ; 4,0)$ & Total Presença & $\begin{array}{l}\text { Indica que não há dúvidas de que o critério está } \\
\text { totalmente presente. }\end{array}$ \\
\hline
\end{tabular}


Quarta Etapa: tratamento dos dados coletados dos especialistas, na avaliação de cada item coletado.

Nesta etapa, foi realizado o tratamento dos dados coletados na avaliação de cada item. O processo de obtenção dos números fuzzy de cada item avaliado levou em conta o grau de importância relativa de cada especialista, obtidos através do preenchimento do QIPE. As notas dos itens foram agregadas para compor a nota de avaliação do processo de gerenciamento.

Os resultados desta pesquisa de campo são apresentados e analisados a seguir em relação aos processos e às áreas de gerência consideradas para a avaliação da qualidade de projetos de software.

\section{Características de Qualidade da Gerência de Projetos de Software}

A pesquisa indicou que gerentes de projetos com pouco conhecimento sobre metodologias de gerenciamento tinham a tendência de subestimar a importância dos processos de gerenciamento, atribuindo notas baixas aos itens do questionário. Neste caso, como o modelo de avaliação da qualidade trabalha ponderando o grau de similaridade entre as respostas analisadas, foi feito o tratamento automático desta situação [2].

A Tabela 3 apresenta os resultados obtidos da avaliação da qualidade dos processos de gerenciamento de projetos de software. Esses resultados levaram em consideração o perfil de cada um dos avaliadores, conforme orienta o modelo de avaliação utilizado.

Para a defuzificação, foi considerado apenas o valor $m$ do número fuzzy normal triangular, representado por $\tilde{N}(a, m, b)$. Assim sendo, pode-se considerar o intervalo de valores $[a, b]$ como sendo o intervalo desejável de qualidade para o processo ou a área de gerência avaliada.

O processo da Verificação do Escopo utiliza documentos produzidos pelos processos de planejamento e pela documentação sobre os produtos e serviços, que serão gerados pelo projeto para realizar medições, testes, verificações de resultados com o objetivo de assegurar que o trabalho realizado esteja em conformidade com os requisitos. Como resultado final, o processo produz a documentação de aceitação formal, pelo cliente, dos produtos gerados. Esse foi o processo de maior grau de importância entre os gerentes de projeto com o valor de 3,64, o que representa $36 \%$ de alta presença e $64 \%$ de total presença.

O processo da Estimativa de Duração das Atividades é responsável pela medição do tempo de realização das atividades em projetos de software, envolvendo a interdependência e o inter-relacionamento entre elas. A importância da estimativa e do acompanhamento do tempo de execução das atividades está relacionada diretamente com os fatores de custo, risco e qualidade do projeto. A pesquisa indicou uma grande preocupação dos gerentes de projetos com o fator tempo. Esse processo ficou em segundo lugar com o grau de importância de 3,33, com $67 \%$ de alta presença e $33 \%$ de total presença.

O processo do Desenvolvimento do Plano do Projeto gera um documento, que consolida as informações necessárias para o gerenciamento dos processos de produção do projeto. Esse documento funciona como um guia para o gerenciamento do projeto. Contém informações específicas sobre escopo, custo, cronograma, fatores de qualidade, riscos e pessoas envolvidas no projeto. $\mathrm{O}$ uso de ferramentas automatizadas para a geração e a atualização dessas informações já é uma realidade e tem se mostrado ideal para o gerenciamento de projetos com equipes geograficamente distribuídas através da internet. Esse 
processo obteve o grau de importância de 3,19. Ficou em terceiro lugar, com $81 \%$ de alta presença e 19\% de total presença.

Tabela 3: Resultados da avaliação do processo de gerenciamento de projetos de software

\begin{tabular}{|c|c|c|}
\hline \multicolumn{2}{|l|}{ Gerência do Escopo } & $\tilde{N}=(\mathbf{2 , 0 8} ; 3,07 ; 3,60)$ \\
\hline Iniciação & INI & $\tilde{N}=(2,13 ; 3,12 ; 3,70)$ \\
\hline Definição do Escopo & DEC & $\tilde{N}=(1,81 ; 2,76 ; 3,43)$ \\
\hline Planejamento do Escopo & PEC & $\tilde{N}=(2,04 ; 3,04 ; 3,65)$ \\
\hline Verificação do Escopo & VEC & $\tilde{N}=(2,64 ; 3,64 ; 3,90)$ \\
\hline Controle das Mudanças no Escopo & CME & $\bar{N}=(1,79 ; 2,79 ; 3,31)$ \\
\hline \multicolumn{2}{|l|}{ Gerência do Prazo } & $\tilde{N}=(1,97 ; 2,91 ; 3,48)$ \\
\hline Definição das Atividades & DAT & $\tilde{N}=(1,88 ; 2,85 ; 3,41)$ \\
\hline Seqûenciamento das Atividades & SAT & $\tilde{N}=(1,96 ; 2,74 ; 3,21)$ \\
\hline Estimativa de Duração das Atividades & EDA & $\tilde{N}=(2,33 ; 3,33 ; 3,88)$ \\
\hline Desenvolvimento do Cronograma & DCR & $\tilde{N}=(1,79 ; 2,75 ; 3,45)$ \\
\hline Controle do Cronograma & CCR & $\tilde{N}=(1,91 ; 2,89 ; 3,47)$ \\
\hline \multicolumn{2}{|l|}{ Gerëncia da Comunicação } & $\tilde{N}=(1,85 ; 2,81 ; 3,49)$ \\
\hline Planejamento da Comunicação & PCM & $\tilde{N}=(1,43 ; 2,33 ; 3,19)$ \\
\hline Disponibilidade de Informações & DIF & $\tilde{N}=(2,08 ; 3,07 ; 3,71)$ \\
\hline Comunicação do Desempenho & $\mathrm{CDE}$ & $\tilde{N}=(1,73 ; 2,73 ; 3,32)$ \\
\hline Fechamento Administrativo & FAD & $\tilde{N}=(2,17 ; 3,10 ; 3,74)$ \\
\hline \multicolumn{2}{|l|}{ Gerëncia da Integração } & $\tilde{N}=(1,86 ; 2,77 ; 3,31)$ \\
\hline Desenvolvimento do Plano do Projeto & DPP & $\tilde{N}=(2,21 ; 3,19 ; 3,55)$ \\
\hline Execução do Plano do Projeto & EPP & $\tilde{N}=(1,88 ; 2,67 ; 3,14)$ \\
\hline Controle das Mudanças no Projeto & CMP & $\tilde{N}=(1,48 ; 2,44 ; 3,23)$ \\
\hline Gerëncia do Custo & & $\tilde{N}=(1,70 ; 2,63 ; 3,37)$ \\
\hline Planejamento dos Recursos & PRC & $\tilde{N}=(1,67 ; 2,61 ; 3,35)$ \\
\hline Estimativa dos Custos & ECU & $\tilde{N}=(1,77 ; 2,73 ; 3,47)$ \\
\hline Orçamentação dos Custos & OCU & $\tilde{N}=(1,76 ; 2,70 ; 3,54)$ \\
\hline Controle dos Custos & $\mathrm{CCU}$ & $\tilde{N}=(1,61 ; 2,49 ; 3,10)$ \\
\hline \multicolumn{2}{|l|}{ Gerëncia da Qualidade } & $\tilde{N}=(1,53 ; 2,42 ; 3,12)$ \\
\hline Planejamento da Qualidade & PQU & $\tilde{N}=(1,80 ; 2,69 ; 3,37)$ \\
\hline Garantia da Qualidade & GQU & $\tilde{N}=(1,76 ; 2,70 ; 3,29)$ \\
\hline Controle da Qualidade & $\mathrm{CQU}$ & $\tilde{N}=(1,03 ; 1,86 ; 2,70)$ \\
\hline \multicolumn{2}{|l|}{ Gerëncia do Risco } & $\tilde{N}=(1,45 ; 2,35 ; 3,11)$ \\
\hline Análise Qualitativa do Risco & AQR1 & $\tilde{N}=(1,72 ; 2,63 ; 3,36)$ \\
\hline Análise Quantitativa do Risco & AQR2 & $\tilde{N}=(1,03 ; 1,87 ; 2,84)$ \\
\hline Planejamento do Gerenciamento do Risco & PGR & $\tilde{N}=(1,78 ; 2,78 ; 3,40)$ \\
\hline Planejamento das Respostas aos Riscos & PRR & $\tilde{N}=(1,56 ; 2,39 ; 3,05)$ \\
\hline Monitoração e Controle de Riscos & MCR & $\tilde{N}=(1,14 ; 2,06 ; 2,91)$ \\
\hline
\end{tabular}


A seguir serão apresentadas as principais conclusões e contribuições deste trabalho, relativas aos processos de gerenciamento de projetos de software.

\section{Conclusão}

Este trabalho demonstrou que projetos de desenvolvimento e de manutenção de software devem ser conduzidos através de processos gerenciais e de produção padronizados. A norma ISO/IEC 12207 e os modelos de maturidade estabelecem a definição de um processo padrão como condição para o estabelecimento de um processo de software disciplinado.

Entre outros resultados, obteve-se que as gerências de escopo, de prazo e comunicação foram consideradas as mais relevantes pelos avaliadores, enquanto que a gerência de risco foi considerada como a de menor relevância. Ficou evidenciado que o desenvolvimento de projetos de software envolve muitos fatores que são difíceis de gerenciar em um ambiente de mudanças constantes. Para minimizar estes problemas, o gerente do projeto tem o importante papel de alinhar os objetivos do projeto com as estratégias corporativas da organização.

Este trabalho teve as seguintes contribuições:

- A proposta de um modelo de avaliação da qualidade da gerência de projetos de software, levando-se em consideração os processos gerenciais abordados no PMBOK, na ISO/IEC 12207, no ISO/IEC TR 15505, na ISO 10006, e na ISO/IEC DTR 16326.

- O levantamento de um padrão de qualidade para gerência de projetos de software e seus respectivos processos gerenciais, através do estabelecimento de um índice de qualidade, segundo o modelo fuzzy de avaliação de qualidade utilizado.

- O mapeamento dos processos do PMBOK com as normas ISO/IEC 12207, ISO 10006 e o ISO/IEC TR 15504.

\section{Referências Bibliográficas}

[1] Belchior, A. D., 1997, Um Modelo Fuzzy para Avaliação da Qualidade de Software, Tese de Doutorado, COPPE/UFRJ.

[2] Branco Jr., E. C., Belchior, A. D., 2001, Processos Gerenciais de Projetos de Software: Uma Abordagem Qualitativa, Workshop de Qualidade de Software, XV SBES, Rio de Janeiro.

[3] Ibbs, C. W., Kwak, Young Hoom, 1997, Measuring project management's return on investment, PM Network, pp. 36-38.

[4] Ibbs, C. W., Kwak, Young-Hoon, 1997, Benchmarking project management organizations, PM Network, pp. 49-53.

[5] Ibbs, C.W., Kwak, Young-Hoon, Calculating Project Management's Return on Investment, forthcoming in Project Management Journal.

[6] INGRAM, T., 2000, How to Turn Computer Problems into Competitive Advantage, Project Management Institute

[7] ISO/IEC DTR 16326, 1999, Software Engineering - Guide for the application of ISO/IEC 12207 to project management.

[8] ISO 9000-3, 1993, Quality Management and Quality Assurance Standards - Part 3: Guidelines for the Application of ISO9001 to the Development, Supply and Maintenance of Software, ISO. 
[9] ISO/IEC TR 15504, 1998, Software Process Assessment, Part 1-9, SPICE Project Organization.

[10] NBR ISO 10006, 2000, Gestão da Qualidade - Diretrizes para a qualidade no gerenciamento de projetos, ABNT, Dezembro.

[11] NBR ISO/IEC 12207, 2000, Tecnologia de Informação - Processos de ciclo de vida de software.

[12] PAULK. M. C. et al., 1993, Chrissis, and M.Bush, Key Practices of the Capability Maturity Model, Version 1.1, Software Engineering Institute, CMU/SEI-93-TR-25, February.

[13] PAULK, M. C. et al, 1993, Capability Maturity Model for Software, version 1.1 Technical Report ,CMU/SEI-93-TR-024,ESC-TR-93-177, February.

[14] Pedryez, W., Gomide, F, 1998, An Introduction to Fuzzy Sets, Analysis and Design, MIT Press, Cambridge, Massachusetts, London.

[15] PMI Standards Committee, 2000, A Guide to the Project Management Body of Knowledge (PMBOK), Philadelphia, PA, USA, PMI Publishing Division.

[16] PMI - Project Management Institute, 2001, http://www.pmi.org, acessado em: Outubro.

[17] Pressman, R. S. P., 2000, Software Engineering - A Practitioner's Approach, Fifth Edition, McGrawHill.

[18] PRINCE, 2001, http://www.prince2.com/, acessado em: Outubro/2001.

[19] Guide to the SWEBOK, 2001, http://www.swebok.org/, acessado em: Outubro/2001.

[20] TONEY, F., POWERS, R., 2000, Best Practices of Project Management Groups in Large Functional Organizations, Project Management Institute.

[21] Zadeh, L., 1998, Fuzzy Logic, IEEE Transaction Comput, Vol 25. 